\title{
A Financial Decision Supporting System Based on Fuzzy Candlestick Patterns
}

\author{
Chiung-Hon Leon Lee* and Alan Liu \\ *Department of Computer Science and Information Engineering, ChungChou Institute of Technology. \\ Department of Electrical Engineering, National Chung Cheng University, Taiwan.
}

\begin{abstract}
A financial decision supporting system based on the fuzzy candlestick pattern is proposed and developed. We model Japanese candlestick patterns by using fuzzy linguistic variables. Japanese candlestick theory is an empirical model of investment decision. The theory assumes that the trend of financial time series could be predicted by identifying specific candlestick patterns in the candlestick charts. In our system, the investment expertise is represented in fuzzy candlestick patterns and can be stored in a pattern base. The investors can make their investment decisions based on the identified candlestick patterns. A knowledge based pattern recognition method for candlestick pattern is implemented in the system, and the investor can edit, validate, and share the imprecise and vague candlestick patterns through the graphical interface of the proposed system.
\end{abstract}

Keywords: financial, decision supporting system, fuzzy candlestick pattern.

\section{Introduction}

Japanese candlestick analysis is one of the most widely used technical analysis techniques and definitely viable and effective for stock and commodity market timing and analysis [1]. Japanese candlestick theory is an empirical model of investment decision. The theory assumes that the trend of financial time series could be predicted by identifying specific candlestick patterns in the candlestick charts. The investors make investment decisions by the identified candlestick patterns.

The advantage of the candlestick theory to investors is that the candlestick chart is visual, and a reversal or continuation candlestick pattern can be easily identified by an experienced investor. There is rich information which exists in the financial time series database, but most of the traditional approaches only scratch the surface of the wealth of knowledge buried in the data. For example, many financial time series prediction approaches only use a single type of value, such as daily closing price, as raw data to construct the forecasting model.

Figure 1 shows different ways to represent the stock trading price during a trading time period. Figure 1(a) indicates a single closing price. Figure 1(b) represents the bar line which contains richer information than 1(a). The data required to produce a standard bar chart consists of the open, high, low, and close prices for the time period under study. The high price and low price refers to the highest price and lowest price during the trading time period. A bar chart consists of vertical lines representing the high to low range in prices for that trading time period. Figure 1(c) illustrates the candlestick line which is similar to the bar line but using a box to makes up the difference between the open and close price.

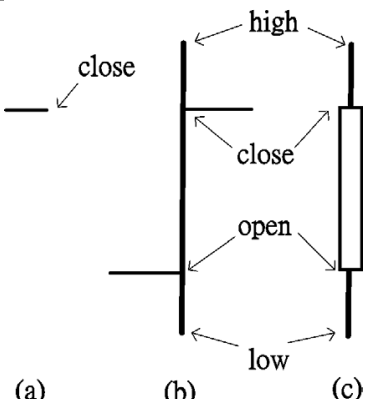

Figure 1. Different ways to represent the stock trading prices.

The disadvantage when applying the candlestick theory are that identifying the candlestick pattern from a large amount of trading data is time consuming and there are no crisp and standard definitions to the candlestick patterns. It needs investment experiences in many years to a human investor to select an effective pattern from a lot of imprecise and vague candlestick patterns. The imprecise and vague definitions of the candlestick patterns also make the automated searching, mining, and processing the candlestick patterns with computer software difficult.

In [2], we solve these problems by using fuzzy set theory [3]. The imprecise and vague candlestick patterns are represented by fuzzy linguistic variables. 
Based on our previous work, in this paper, we propose and develop a fuzzy candlestick pattern based decision supporting system to help the user to extract pattern from the historical financial time series, edit extracted patterns, store patterns, and using the stored patterns to give investment suggestions for the investors. To the investors, the system can also be used as a platform to learn and share the investment expertise, because the investment expertise is represented in the fuzzy candlestick patterns and can be stored in the database.

The paper is organized as follows. In Section 2, how to represent candlestick patterns in fuzzy linguistic variable is introduced. Section 3 describes the proposed system. Finally, Section 4 provides the conclusion of this paper.

\section{Knowledge Representation}

How to transfer financial time series into candlestick chart and how to represent candlestick pattern in fuzzy linguistic variables are two important problems when constructing the candlestick pattern based investment decision supporting system.

\subsection{Candlestick chart}

Figure 2 shows an example of the daily candlestick chart for the stock market. Daily open, close, high, and low prices are recorded in the candlestick lines form d1 to $\mathrm{d} 10$.

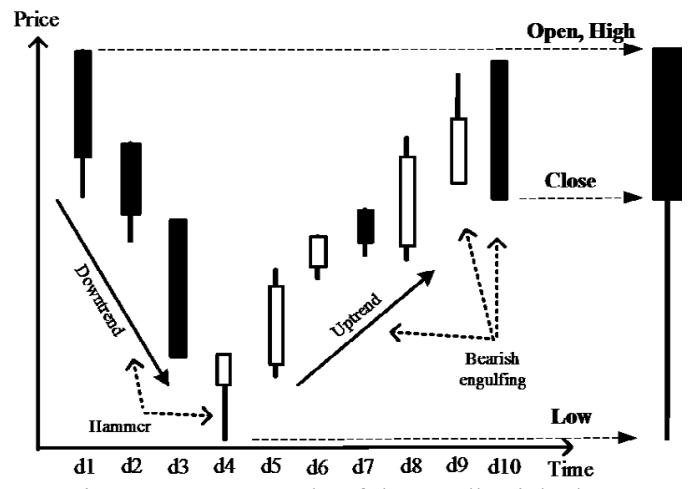

Figure 2. An example of the candlestick chart.

On the day $\mathrm{d} 3$, the price closes at a lowest price and continues the downtrend from $\mathrm{d} 1$ to $\mathrm{d} 2$. On the day $\mathrm{d} 4$, the opening price is lower than previous closing price, but the price closes at the highest price and leaves a long lower shadow. This situation might be interpreted by an experienced investor as the candlestick line on the day from $\mathrm{d} 1$ to $\mathrm{d} 3$ reflecting a downtrend of the stock price, because there are many investors who want to sell the stock, making the closing price much lower than the opening price. However, the downtrend might reverse itself on the day $\mathrm{d} 4$, because there might be investors wanting to buy the stock in the trading period that makes the price close at the highest price and leave a long lower shadow. In other word, the candlestick lines at $\mathrm{d} 3$ and $\mathrm{d} 4$ can be interpreted that the downtrend is bouncing back.

At $\mathrm{d} 9$, the closing price is higher than the opening price, but the long upper shadow indicates that there are some investors start to sell their stocks. At d10, the opening price is much higher than the previous closing price, but it closes at lowest price and lowers than the close price on previous day. The lines at $\mathrm{d} 9$ and $\mathrm{d} 10$ can represent a reverse, because the downtrend is broken at $\mathrm{d} 10$.

A candlestick pattern is composed by one or more candlestick lines and the trend before the pattern. By the trading experience, the investor tries to identify the candlestick patterns to help themselves to make the investment decisions such as to buy, sell, or hold the stock. There are many existing defined candlestick patterns which are widely used by the investors [1]. In Figure 2, the candlestick line on $\mathrm{d} 4$ and the trend formed by $\mathrm{d} 1, \mathrm{~d} 2$, and $\mathrm{d} 3$ are defined as a pattern which is called Hammer to represent the downtrend is reversed. Another pattern called Bearish engulfing is also illustrated in Figure 2 and is composed by a uptrend and the candlestick lines on $\mathrm{d} 9$ and $\mathrm{d} 10$.

\subsection{Fuzzy candlestick patterns}

How to represent a candlestick line and how represent the relationship between two continues candlesticks lines are to major problems when represent a candlestick pattern. A candlestick line is represented by six parts: open style, upper shadow, body, body color, lower shadow, and close style.

Figure 3 shows an example of the fuzzy membership function $\mu(x)$ of the linguistic variables for representing the body and shadows length of a candlestick line. Four fuzzy linguistic variables EQUAL, SHORT, MIDDLE, and LONG are defined. The range of body and shadow length is set to 0 to 14 percent of the fluctuation of stock price. It is up to the system designer to change fuzzy sets and the range of the lengths to fit the needs of different investment targets.

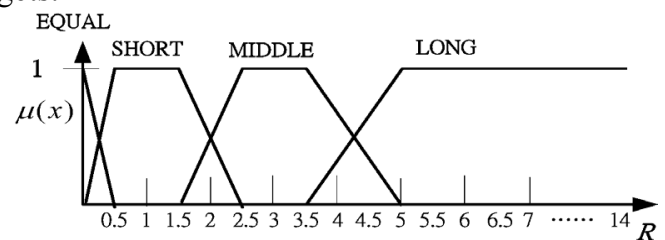

Figure 3 . The fuzzy sets of the length of the body and shadows.

Figure 4 shows the membership function of the linguistic variables of the open style and close style. The candlestick line in the bottom of Figure 4 is the 
candlestick line of previous trading time. The unit of $\mathrm{X}$ axis is the trading prices of previous day and the unit of $\mathrm{Y}$ axis is the possibility values of the membership function.

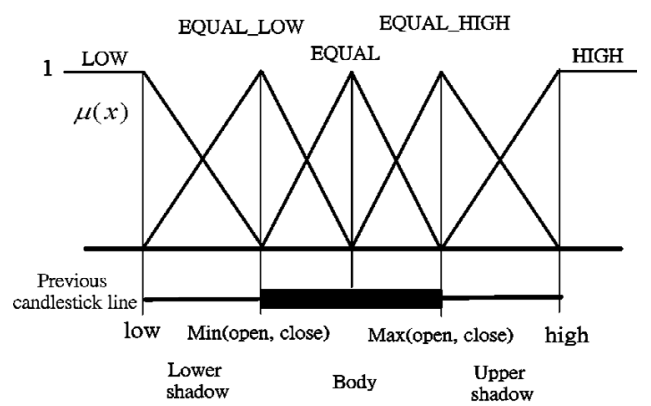

Figure 4. The fuzzy sets of the open style and close style.

Five linguistic variables are defined to represent the open style relationships: OPEN LOW, OPEN EQUAL_LOW, OPEN EQUAL, OPEN EQUAL_HIGH, and OPEN HIGH, and five linguistic variables are defined to represent the close style relationships: CLOSE LOW, CLOSE EQUAL_LOW, CLOSE EQUAL, CLOSE EQUAL_HIGH, and CLOSE HIGH.

Table 1 shows a fuzzy candlestick pattern example which demonstrates a possible way to represent the Bearish Engulfing candlestick pattern, and other candlestick patterns can be defined in the same way. The previous trend defined here is a crisp rule such as "down $15 \%$ in recent 10 days" to represent a downtrend or "up $15 \%$ in recent 10 days" to represent an uptrend.

Table 1: An example of the fuzzy candle pattern.

\begin{tabular}{|c|c|}
\hline Pattern description part & \\
\hline $\begin{array}{lll}\text { Pattern } & \text { name: } & \text { Bearish } \\
\text { Engulfing } & & \\
\end{array}$ & Confirmation suggest: Suggest \\
\hline Previous trend: Uptrend & \multirow{2}{*}{$\begin{array}{l}\text { Confirmation information: } \\
\text { The open price after the pattern } \\
\text { should not be higher than the } \\
\text { open price of candle line } 0 \text {. }\end{array}$} \\
\hline $\begin{array}{l}\text { Candle lines: } \\
\text { Candle line 0: } \\
\text { Open style: OPEN HIGH } \\
\text { Close style: CLOSE LOW }\end{array}$ & \\
\hline Upper shadow: null & \multirow{4}{*}{$\begin{array}{l}\text { Recognition rule: } \\
\text { 1. A definite downtrend must be } \\
\text { underway. } \\
\text { 2. The second day's body must } \\
\text { completely engulf the prior day's } \\
\text { body. } \\
\text { 3. The first day's color should } \\
\text { reflect the trend: black for a } \\
\text { downtrend and white... }\end{array}$} \\
\hline Body: ABOVI & \\
\hline $\begin{array}{l}\text { Body color: } \mathrm{BLACK} \\
\text { Lower shadow: null }\end{array}$ & \\
\hline $\begin{array}{l}\text { Candle line 1: } \\
\text { Open style: ABOVE OPEN } \\
\text { EQUAL_LOW } \\
\text { Close style: CLOSE HIGH } \\
\text { Upper shadow: null }\end{array}$ & \\
\hline Body: ABOVE SHORT & \multirow{3}{*}{$\begin{array}{l}\text { Pattern explanation: } \\
\text { The first day of the } \\
\text { Engulfing pattern has a small } \\
\text { body and the second day has a } \\
\text { long real body. Because the } \\
\text { second day's move.... }\end{array}$} \\
\hline $\begin{array}{l}\text { Body color: WHITE } \\
\text { Lower shadow: null }\end{array}$ & \\
\hline & \\
\hline
\end{tabular}

The fuzzy modifiers are used to further enhance the flexibility of the linguistic variables in fuzzy candlestick patterns. Modifiers used in phrases such as
$B E L O W$ or $A B O V E$ change the shape of the modified fuzzy sets.

\subsection{Fuzzy pattern recognition}

Since the patterns have been defined by the investor, the defined patterns can be easily transferred into fuzzy rules. For example, the Bearish Engulfing pattern can be transferred as following fuzzy rule.

IF trend $=$ UP TREND,

AND line0.open_style $=$ OPEN HIGH,

AND line0.close_style $=$ CLOSE LOW,

AND line 0. body $=$ ABOVE MIDDLE,

AND line 0. body_color $=$ BLACK,

AND line1.open_style = ABOVE OPEN

EQUAL_LOW,

AND line1.close_style $=$ CLOSE HIGH,

AND line1.body $=$ ABOVE SHORT,

AND line1.body color $=$ WHITE,

THEN the pattern $=$ BEARISH ENGULFING.

A pattern recognition rule consists of the crisp part and the fuzzy part. The crisp part includes the previous trend of the pattern and the body color. The others of the rule are the fuzzy part such as the body and shadow length and the open and close style. From observation, well arranged identification rule will reduce the pattern recognition processing time.

Comparing with the processing time of the fuzzy part, the crisp part takes less processing time. For example, the body color includes three possibilities: BLACK, WHITE, and CROSS. For judging the value of the body color, the pattern recognition module only needs to compare the value of open price and close price. The pattern identifying time can be reduced if the judgment of the crisp part is placed before the process of the fuzzy part.

\subsection{Mining patterns}

Since the candlestick theory assumes that the trading intention of the investor can be reflect in the candlestick chart, the forecasting problem for the investor becomes how to find the candlestick patterns when the uptrend is returned or the downtrend is bouncing back, in other word, how to find the reversal patterns when the uptrend start becomes downtrend or the downtrend becomes uptrend.

The candlestick patterns mining process is illustrated in Figure 5. First, the stock prices time series is acquired from the database and transfer into fuzzy candlestick patterns. There might be more than one fuzzy set matched for a single crisp value when finding the value of the linguistic variable. For disembogues, the fuzzy set with biggest membership value will be selected. The amount candlestick lines which to compose the candlestick pattern are assigned by the user. 
Based on the following trend, the ID3 classification algorithm [4] is used to classify the fuzzy candlestick patterns, because it is a method for approximating discrete-valued functions, robust to noisy data, and capable of learning disjunctive expressions. We use the algorithm to filter the attributes is less important to the following trend.

Because the investor is interested in the reversal patterns, the pattern with the previous trend is STRONG BEARISH or EXTREME BEARISH and the following trend is STRONG BULLISH or EXTREME BULLISH will be selected as the candidate patterns for prediction. The mined pattern can be easily transferred into fuzzy rules like follows.

IF the previous trend $=$ STRONG BEARISH,

AND Line 1 body $=$ EQUAL WHITE,

AND Line 0 body $=$ MIDDLE BLACK,

THEN the following trend $=$ STRONG BULLISH.

Finally, using the simple mechanism of symbolic matching process, the investor can validate the efficiency of the selected patterns and add comments for the mined patterns.

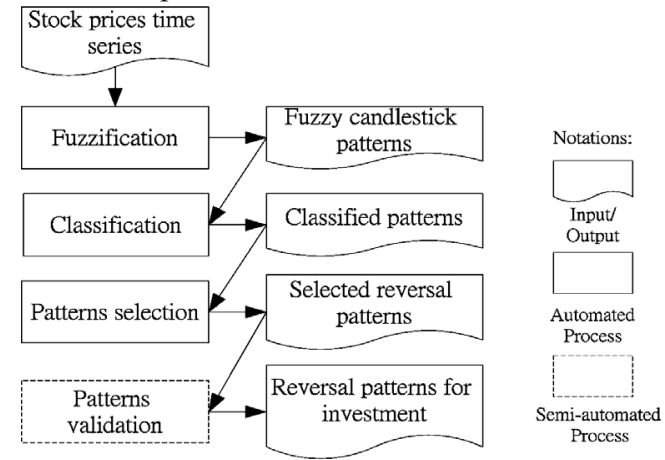

Figure 5. The process of mining candlestick patterns.

\section{Implementation}

The system in this paper is a continuation to our previous work of Candlestick Tutor (CT) [5]. Two kinds of users are identified, the pattern editor and the investor. The requirements posted by the pattern editor are defining, editing, and storing the candlestick patterns. The requirement raised by the investor is recognizing the patterns from the stock trading information.

For fulfilling the user's requirements, the system is composed by five modules, a graphical user interface (GUI), a pattern authoring tool, a pattern validation tool, an information management module, and a pattern recognition module. The user edits the candlestick patterns in the pattern authoring tool, validates the patterns by using the validation tool, stores and retrieves the defined patterns to the database via the information management module, interacts with the system and observes the candlestick patterns through the GUI. The pattern recognition module performs the fuzzy candlestick pattern recognition process to retrieve the user interested patterns from the stock information database.

We also designed an information agent to collect the financial data. After each trading day, the information agent connects to a website which provides the stock information, such as Yahoo, acquires and parses the stock information from Web pages, and stores the acquired data to the stock information database automatically. The information agent also transfers the trading prices and volume of the stock to the technical indexes such as RSI, KD, and MACD etc. When all of the stock information have been extracted from the Web pages and stored to the stock information database, the information agent queries the database to retrieve the previous technical index and stock prices data to calculate the new technical index data and store the data to the stock information database for future usage. The investor can use technical index information to enhance the efficiency of candlestick patterns.

\section{Conclusion}

The fuzzy candlestick patterns carry rich information and can be used to increase the efficiency of the data mining, machine learning, and pattern recognition models. Pattern construction and recognition procedures is introduced and implemented in a system prototype to illustrate the usage of the fuzzy candlestick patterns. Moreover, investors can save and share their investment experience. By reusing and modifying the stored candlestick pattern information, the investor can also increase the efficiency of their investing strategies.

\section{References}

[1] G. L. Morris, Candlestick Charting Explained: Timeless Techniques for Trading Stocks and Futures 2nd edition, McGraw-Hill Trade, 1995.

[2] C.H.L Lee, A. Liu, and Wen-Sung Chen, "Pattern Discovery of Fuzzy Time Series for Financial Prediction," IEEE Trans. on Knowledge and Data Engineering, Vol. 18, no. 5, May, 2006, pp. 613-625.

[3] G.J. Klir, and B. Yuan, Fuzzy sets and fuzzy logic theory and application, Prentice Hall, Upper Saddle River, NJ, 1995.

[4] Ian H.W. and Eide F., Data Mining - practical machine learning tools and techniques with Java implementations, Morgan Kaufmann, San Francisco, 2000.

[5] C.H.L Lee, W. Chen, and A. Liu, "An Implementation of Knowledge Based Pattern Recognition for Finicial Prediction," in proc. 2004 CIS-RAM, Singapor, pp.218-223. 